\title{
Consumo de drogas y adherencia a la terapia antirretroviral de pacientes en programa de mantenimiento con metadona
}

\author{
Lucía Ladero Martín*; Santos Orejudo Hernández**; José Antonio Carrobles Isabel***; \\ Carmen Malo Aznar**** \\ ${ }^{*}$ CAID Torrejón de Ardoz \\ **Departamento de Psicología y Sociología. Universidad de Zaragoza \\ ***Departamento de Psicología Biológica y de la Salud. Universidad Autónoma de Madrid \\ *** Servicio de Educación de Huesca. Diputación General de Aragón
}

Enviar correspondencia a:

Santos Orejudo Hernández. Facultad de Educación - Universidad de Zaragoza. C/ San Juan Bosco, 7. 50009 - Zaragoza. sorejudo@unizar.es

Recibido: 14 de Octubre de 2004 Aceptado: 7 de Febrero de 2005.

\section{RESUMEN}

En la actualidad la TARGA (Terapia Antirretroviral de Gran Actividad) constituye el estándar de tratamiento frente a la infección por el VIH, siendo la adherencia o el cumplimiento con la misma uno de los criterios de éxito en la reducción de la carga viral. Los problemas de falta de adhesión al tratamiento están presentes en gran medida en el grupo de pacientes con antecedentes de consumo de drogas, por lo que conocer los factores asociados a la misma puede ser gran utilidad para mejorar las intervenciones en este campo.

En este trabajo analizamos la relación existente entre la adhesión al tratamiento antirretroviral y el consumo de drogas en una muestra de 100 sujetos $\mathrm{VIH}+$ incluidos en un programa de mantenimiento con metadona en Madrid. Se han clasificado a los sujetos en adherentes y no adherentes según intervalos temporales teniendo en cuenta el momento de toma de medicación referido (día, semana y mes anterior) y el grado de cumplimiento (alta y baja exigencia), analizándose a continuación las diferencias que presentaban en consumo. Los resultados indican que nuestra muestra presentan niveles de adherencia similares a otras poblaciones con enfermedades crónicas, y que el hecho de consumir drogas tiene una relación estadísticamente significativa con el no cumplimiento de la TARGA. Cuando se usan los indicadores de consumo para clasificar a los sujetos en adherentes y no adherentes, encontramos que el policonsumo de heroína, cocaína y benzodiacepinas aparece como principal predictor de no adhesión a la terapia antirretroviral.

Palabras clave: Adherencia, TARGA, consumo de drogas, tratamiento con metadona, drogodependientes.

\section{ABSTRACT}

Nowadays, HAART (Highly Active Antiretroviral Therapies) is the standard treatment for HIV infection, and adherence or compliance with it is one of the successful factors in reducing viral load. The lack of adherence to the treatment is present to a large extent in the patient population with a history of drug use. This is why an understanding of the associated factors could be of great importance in improving interventions in this field.

In this work, the relationship between adherence to HAART and drug use was studied with a sample of 100 HIV-positive patients undergoing a methadone maintenance programme in Madrid (Spain). The sample was divided into adherents and nonadherents, in accordance with medication intake (day, week and previous month) and the degree of compliance (high and low). We then proceeded to analyse the presented differences in drug use.

The results indicate that our sample presents adherence levels similar to other populations with chronic diseases and that taking drugs has a statistically significant association with noncompliance with HAART. When use indicators are employed in classifying subjects as adherents or non-adherents, we find that the multidrug use of heroin, cocaine and tranquillizers appears as the principal predictor of non-adherence to antiretroviral therapy.

Key words: Adherence, HAART, drug use, methadone maintenance programme, drug addicts.

\section{INTRODUCCIÓN}

A ntes de la introducción de la TARGA (Terapia Antirretroviral de Gran Actividad), los casos de SIDA tenían un período de supervivencia de 18 meses, como máximo, y, el período asintomático de la infección oscilaba alrededor de los 8 años'. Con la TAR-
GA, estos períodos han aumentado considerablemente, ya que los inhibidores de la proteasa, por ejemplo, pueden llegar a disminuir en un 99,9\% el $n^{\circ}$ de copias de ARN del virus $\mathrm{VIH}-\mathbf{1}^{2,3}$.

Los tratamientos farmacológicos actuales para el VIH se clasifican en tres categorías: Inhibidores de la 
proteasa, análogos de nucleósidos inhibidores de la transcriptasa inversa e inhibidores de la transcriptasa inversa no nucleósidos ${ }^{4,5}$. El uso combinado de estos tres tipos de fármacos en las fases iniciales de la infección, cuando la cantidad de virus es escasa, hace que el tratamiento sea más eficaz.

En el caso del VIH-SIDA, los avances en el tratamiento farmacológico y en los medios de diagnóstico no siempre han acarreado reducciones en la mortalidad y morbilidad de los pacientes. Uno de los principales factores responsables de esta situación es la falta de adherencia o cumplimiento con los tratamientos, ya que, la eficacia de un tratamiento depende en gran medida que el paciente siga las indicaciones del médico ${ }^{6}$.

Lo que ocurre con la falta de adherencia de la medicación antirretroviral, a diferencia de cualquier otra enfermedad crónica, es que las consecuencias afectan tanto al paciente como a la sociedad, ya que el paciente empeora en su estado de salud, aparecen resistencias, se reducen sus posibilidades terapéuticas futuras, aumenta la carga viral, disminuyen los linfocitos $C_{4}$ y aumentan los riesgos de transmisión de cepas resistentes del virus ${ }^{7}$.

La conducta de cumplimiento se puede definir como "la medida en que la conducta de una persona coincide con el consejo médico o sanitario" 8 . Se estima que una media de un $40 \%$ de los pacientes no cumple adecuadamente las recomendaciones terapéuticas de sus médicos, lo que supone dos de cada cinco pacientes ${ }^{9}$. Naturalmente, hay diferencias según factores muy diversos. Los diferentes factores que influyen en la conducta de cumplimiento del tratamiento médico pueden clasificarse en cuatro grandes grupos: a) interacción con el profesional sanitario, b) referidos al régimen terapéutico: tipo, complejidad, exigencia comportamental, duración, costos-beneficios, eficacia, efectos secundarios, contexto social, c) referidos a la enfermedad de que se trate: aguda o crónica, y d) aspectos psicosociales del paciente: cognitivos, emocionales, sociales, apoyo social ${ }^{10}$.

El hecho de que el tratamiento del VIH-SIDA implique generalmente la combinación de muchos tipos de fármacos y con pautas rigurosas de frecuencia, momento de toma, etc., durante un período de tiempo indefinido y con efectos secundarios añadidos, hace que sea más probable el incumplimiento del tratamiento por parte de estos pacientes.

Los pacientes con VIH-SIDA con antecedentes de consumo de drogas o que están en programas de mantenimiento con metadona presentan menores tasas de adherencia11,12,13. Aunque los datos de cumplimiento de tratamiento antirretroviral recogidos en la literatura son escasos, diversos estudios objetivan una tasa de incumplimiento que oscila entre un 15 y un $37 \%{ }^{14,15}$. De cualquier forma, es difícil comparar entre los diferentes trabajos debido a que las muestras, el método, el procedimiento, etc. no siempre es coincidente. Por ejemplo, en unos estudios los pacientes tienen prescrita monoterapia y en otros biterapia. Las muestras son muy diversas, en unos casos grandes grupos y en otros pequeños, y con todo tipo de pacientes, tanto con antecedentes de consumo de drogas como sin ellos.

Aunque está constatado la menor adherencia a los fármacos antirretrovirales de los sujetos VIH-SIDA en programas de metadona, son menos conocidos los factores que influyen en la adherencia dentro de los mismos. En algunos estudios, la edad, el consumo de alcohol y otras drogas o la presencia de eventos vitales negativos se asocia con una menor adherencia ${ }^{16}$.

El objetivo principal de este trabajo, profundizando en las variables determinantes de la adhesión terapéutica en los tratamientos antirretrovirales de pacientes en programas de mantenimiento con metadona (PMM), consiste en poner a prueba la hipótesis de que el consumo de drogas influye en la adherencia. Para ello estudiaremos las diferencias encontradas en usuarios adherentes y no adherentes en una muestra de usuarios de la Comunidad de Madrid. Este trabajo resulta especialmente relevante si tenemos en cuenta que en nuestro país, y a diferencia de lo que ocurre en el resto de Europa, existe un predominio mayor de casos VIH-SIDA que han sido o son usuarios de drogas por vía parenteral (UDVP).

\section{MÉTODOS}

\section{Sujetos}

El programa de mantenimiento con metadona del CAID de Torrejón de Ardoz cuenta con 250 sujetos en total, tanto seropositivos como no seropositivos. De estos se seleccionaron todos los sujetos que cumplían los criterios de inclusión en la investigación (ser mayores de 18 años, portadores de VIH, estar en programa de mantenimiento con metadona y tener una prescripción facultativa de antirretrovirales) y ninguna de las condiciones de exclusión (no aceptar el consentimiento informado, no presentarse físicamente a la cita con el investigador, encontrarse durante el periodo de recogida de datos hospitalizado, encamado o preso).

De los sujetos del CAID de Torrejón se seleccionaron los 83 que había en programa en el momento de la evaluación y, posteriormente, se completó la muestra hasta alcanzar un total de 100 con otros 17 sujetos del CAID de San Blas que cumplían con los 
criterios señalados anteriormente. Estos últimos, fueron alistados cuando acudían a recoger sus dosis de metadona al Centro, siguiendo en este caso, un orden de llegada.

La política de los programas de mantenimiento con metadona de ambos centros es de baja umbral, no exigiéndose la abstinencia como condición necesaria para la permanencia en el mismo. El programa se inició en 1995, por lo que hay sujetos que pueden estar en el mismo desde esta fecha. Se facilita la normalización con dosis para casa (semanales y tres veces por semana), quedando la recogida diaria únicamente para los pacientes que inician programa. A los usuarios se les oferta la posibilidad de recoger sus dosis en distintos puntos de dispensación: en el centro, en el Metabús (servicio móvil de dispensación de metadona de la CAM) o en algunas farmacias concertadas.

Todos los usuarios hacen controles toxicológicos, penalizándose la falta a los mismos (entre uno y dos por semana) con pérdidas temporales de la dosis para casa. El equipo de tratamiento está compuesto por un médico, un trabajador social, un educador y un psicólogo. Se presta especial atención al estado de salud del sujeto, por lo que se incluye una intervención psicoeducativa con los usuarios infectados por VIH.

\section{Procedimiento}

La recogida de datos se hizo entre los meses de Abril, Mayo y Junio de 2003. Para la realización del estudio se tomó el listado de todos los usuarios que estaban en el programa de mantenimiento con metadona independientemente de cual fuera el servicio que les dispensara sus dosis y se seleccionaron aquellos sujetos que fueran HIV+. Se consultaron las historias clínicas para comprobar que en algún momento habían iniciado algún periodo de tratamiento con antirretrovirales, independientemente de que continuara con él o no. Una vez recogidos estos datos se les citaba con el entrevistador y se les explicaba las características del estudio invitándoles a participar en él. Todos debían firmar el consentimiento informado y en el transcurso de esta misma cita se les pasaba un cuestionario de evaluación diseñado ad hoc para esta investigación. Las datos fueron recogidos por el entrevistador de modo que se acortara el tiempo, ente $15 \mathrm{y}$ 20 minutos, y que no cansara a los sujetos.

Los datos referentes al estado clínico e inmunológico del sujeto se tomaron de su historia clínica en el CAID y, aunque el seguimiento se lleva a cabo en el Hospital Príncipe de Asturias de Alcalá de Henares o en el Hospital Ramón y Cajal de Madrid, los resultados de los análisis de la carga viral y los linfocitos $\mathrm{CD}_{4}$ fueron aportados por los sujetos o, con el consentimiento del paciente, solicitados a la Unidad de Infecciosas del propio hospital.
La historia de tratamientos previos, la historia de consumo y la frecuencia de asistencia al centro fueron recogidos del expediente de los sujetos en el CAID. Los resultados de los controles toxicológicos se cumplimentaron una vez que el servicio de toxicología de la CAM los enviaba (más o menos una semana después). Una vez recogidos los datos, se tabularon con el programa estadístico SPSS para su posterior análisis estadístico.

\section{Variables del estudio}

Las variables que se han incluido en este estudio son las siguientes:

a) Variables sociodemográficas:

- Edad, sexo, nivel socioeconómico, nivel de estudios, situación laboral, convivencia actual, situación legal anterior y situación legal actual.

b) Variables clínicas y de adhesión a la TARGA

- Estadío CDC, carga viral y linfocitos $C_{4}$.

- Adhesión ayer, adhesión en la última semana y adhesión en el último mes. Los valores adoptados en este caso se pueden observar en las figuras 4-6.

c) Variables relacionadas con el consumo de drogas

- Tiempo de dependencia a opiáceos (expresado en años), tiempo de consumo de cocaína y tiempo de consumo de benzodiacepinas.

- Analítica de orina para detectar consumo de heroína, cocaína, cannabis, benzodiacepinas y metadona.

- Fecha de entrada en el Programa de Mantenimiento con Metadona (PMM), número de veces que ha estado en tratamiento para el problema de drogodependencias (anteriores al PMM), tiempo de permanencia en los tratamientos anteriores al PMM (expresado en meses) y frecuencia de asistencia al centro.

\section{Análisis estadístico}

El análisis de datos se ha hecho en tres niveles. En el primero se han obtenido los valores descriptivos y las frecuencias de las variables incluidas en el estudio. Sobre estas se han realizado algunas transformaciones para facilitar el resto de análisis. Así, se han creado distintos indicadores de adherencia (adherencia semanal y mensual de alta y baja exigencia), incluyendo en la categoría de alta exigencia únicamente a los sujetos que cumplen con la medicación prescrita 
todos los días, mientras que en la de baja exigencia se les añaden los que muestran buenos niveles de adherencia pero que no cumplen perfectamente, esto es, los que toman correctamente la medicación entre 6-4 días por semana y los que toman casi todos los días del mes.

Con las variables de consumo también se han hecho transformaciones con la finalidad de poder obtener un indicador de policonsumo. Así, se ha creado una nueva variable sumatorio de consumo de heroína, cocaína y benzodiacepinas (policonsumo a partir de aquí) que, como su nombre indica, se forma sumando el número de positivos a estas drogas en la muestra de orina del paciente. No se ha incluido la cannabis, ya que tras un análisis factorial, se ha comprobado que esta variable presenta una baja comunalidad con el resto.

El segundo nivel de análisis corresponde al estudio bivariable entre la adhesión al tratamiento antirretroviral y el resto de variables consideradas. Para las variables nominales se han analizado las tablas de contingencia $\left(\chi^{2}\right)$ entre los indicadores de adherencia elaborados y el resto de variables cualitativas del estudio. Para las variables cuantitativas, la opción elegida ha sido el análisis de varianza, tomando la adherencia como variable independiente.

Por último, en el tercer nivel hemos introducido el análisis multivariable para tratar de averiguar la contribución conjunta de las distintas variables a la adhesión. Para ello hemos elegido la regresión logística que a partir de una ecuación formada por distintos predictores permite clasificar a los sujetos en dos categorías y comparar esta clasificación estimada con la pertenencia real de cada sujeto. El método de inclusión de variables predictoras en la ecuación ha sido el steep-wise hacia delante, especialmente útil para eliminar variables predictoras que covarían entre ellas, como ocurre en nuestro caso con los distintos indicadores de consumo. Dado que la muestra es reducida, hemos incluido como predictores únicamente aquellas variables que en los análisis anteriores han mostrado alguna relación con la adhesión: situación legal actual, analíticas de consumo, autoinforme de consumos y sumatorio de consumo.

\section{RESULTADOS}

\section{Resultados descriptivos}

Características sociodemográficas de la muestra

La muestra está compuesta por 100 sujetos, la mayoría hombres (80\%), con estudios primarios (92\%), un nivel socioeconómico bajo (71\%) y una media de edad de 37,01 (D.T. 5,32) años, con edades comprendidas entre los 20 y los 49 años. El 40\% son pensionistas, el $34 \%$ trabajadores eventuales, el $22 \% \%$, se dedica a actividades marginales, y un $4 \%$ son trabajadores fijos. Más de la mitad (56\%) vive con su familia de origen, un $38 \%$ lo hace con su pareja o su propia familia (38\%). El 77\% de los sujetos han estado encarcelados en algún momento de su vida, aunque en el momento de la recogida de la información, sólo el 36\% tenía algún proceso judicial pendiente, mientras que un $11 \%$ estaba en libertad condicional (cumplimiento de condena con asistencia obligada al CAID). El resto, el 53\%, no tenía nada pendiente en el momento de realizar esta investigación.

Características clínicas de la muestra y adhesión a la TARGA.

El mayor porcentaje de los sujetos de la muestra se encuentran en los estadíos B y C de la infección por $\mathrm{VIH}$, con carga viral superior a 50 copias por microlitro y con menos de 200 linfocitos $\mathrm{CD}_{4}$ (figuras 1-3).

\section{Figura 1: Distribución según Carga Viral}

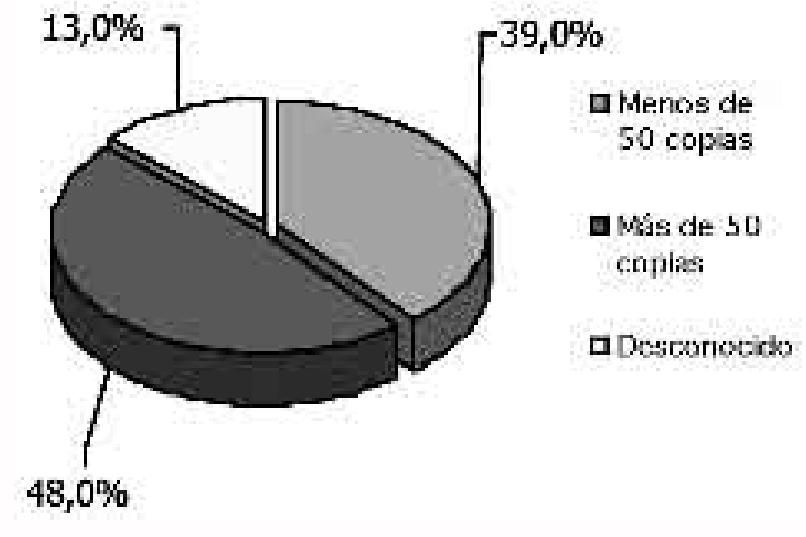

Figura 2: Distribución según estadío CDC

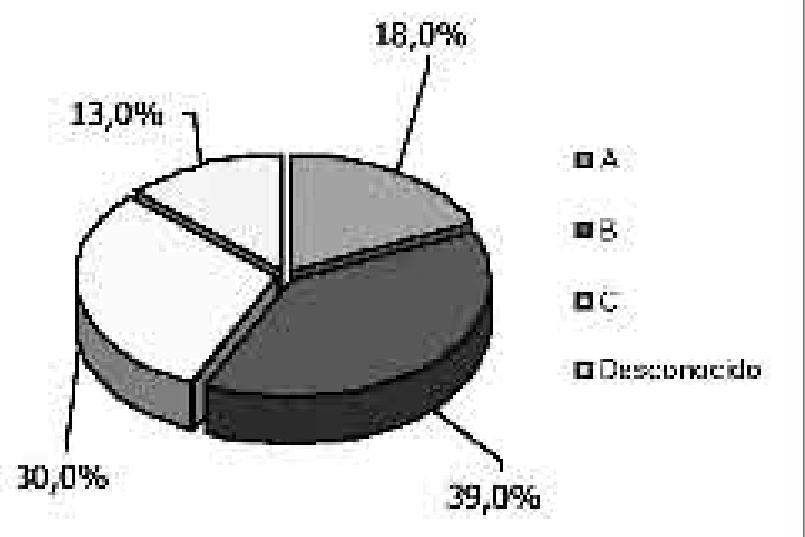


Figura 3: Distribución según Linfocitos $\mathrm{CD}_{4}$

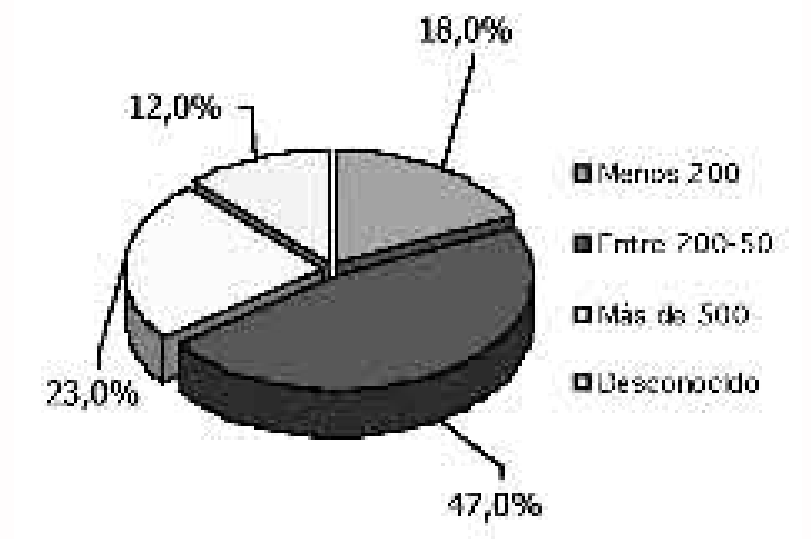

En cuanto a la adhesión al tratamiento antirretroviral, el 61,0\% (figura 4) realizó el día anterior la toma correcta (casos que para el resto de análisis formarán la categoría adhrencia ayer), el 58.0\% (figura 5) lo hizo a lo largo de toda la semana (casos del criterio semana alta exigencia), y el $43 \%$ (figura 6) lo hizo a largo de todo el mes (casos del criterio mes alta exigencia). Para la semana hay también un $6 \%$ de sujetos que muestran buenos niveles de adherencia pero que no cumplen perfectamente, esto es, toman correctamente la medicación entre 6-4 días por semana (figura 5),

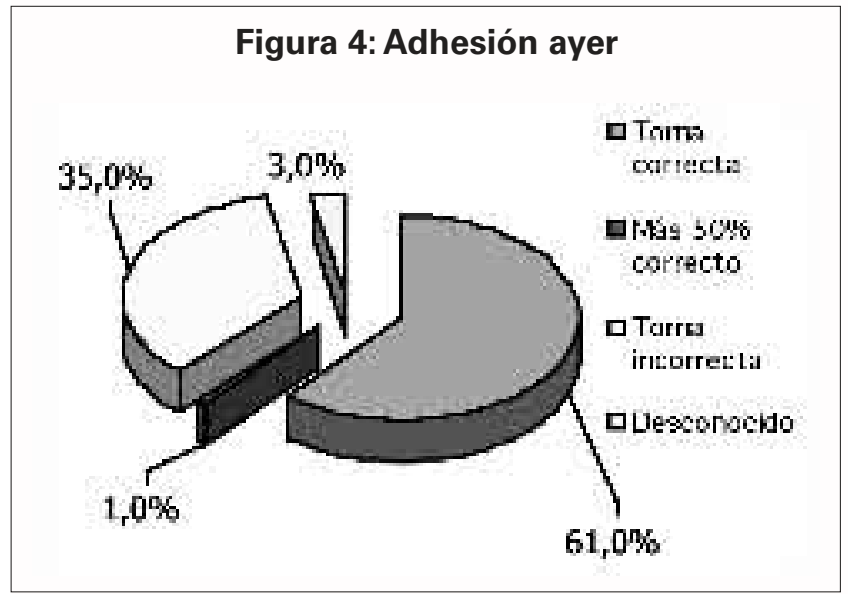

Figura 5: Adhesión última semana

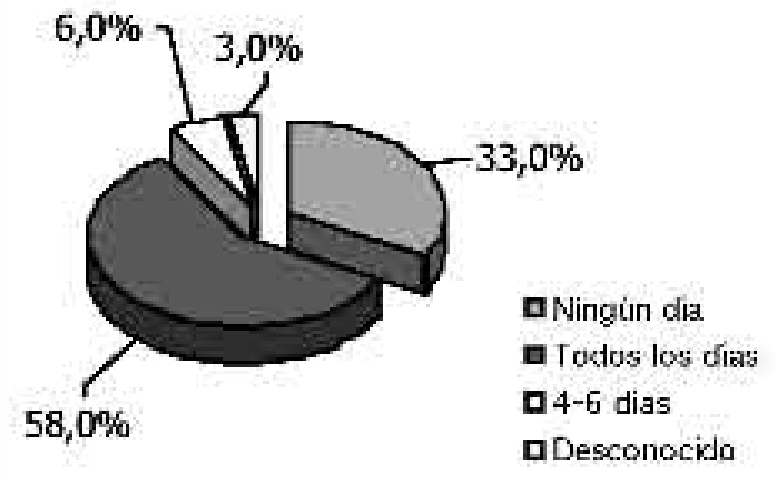

lo que hace un total de $64 \%$ de casos en el criterio de cumplimiento semanal de baja exigencia. Para el mes, hay otro $20,0 \%$ que toman casi todos los días del mes (figura 6), lo que da un total de $63 \%$ de casos en el criterio mensual de baja exigencia.

\section{Figura 6: Adhesión último mes}

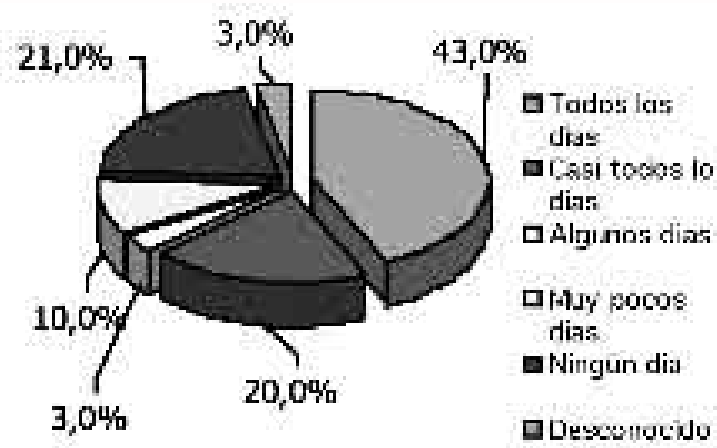

Variables relacionadas con el consumo de drogas.

Para el caso del consumo de heroína, los sujetos de la muestra tienen una historia de 18,12 (D.T. 5,42) años de consumo. Para el resto de drogas, estos valores son menores. La cocaína presenta una historia de consumo de 15,80 años (D.T. 5,90), mientras que el consumo de benzodiacepinas se extiende a 13,91 años de media (D.T. 6,40). Antes de iniciar el programa de mantenimiento con metadona, los usuarios de nuestra muestra habían iniciado una media de 4,23 (D.T. 3,25$)$ tratamientos relacionados con las drogas, en los que habían permanecido una media de 17,46 meses (D.T. 25,40). La estancia media en el programa de metadona se sitúa en los 6 años y 5 meses (Diciembre de 1997), con un intervalo que va desde los sujetos que iniciaron el programa en octubre de 1995 hasta los sujetos que sólo llevan un mes (marzo 2003).

Los sujetos de nuestra muestra consumen distintas drogas y con distinta frecuencia. En las muestras de orina aparecen resultados positivos para heroína en el $19 \%$ de los casos, para cocaína en el $32 \%$, en el $51 \%$ para cannabis y en el $54 \%$ para benzodiacepinas (figura 7). En cuanto al policonsumo, el $36 \%$ de los casos no presenta ningún positivo, otro $36 \%$ da positivo en alguna de estas tres drogas, el $15 \%$ en dos y el $13 \%$ en las tres. Estos porcentajes son muy parecidos a los autoinformes de consumo (figuras 8 , $9,10,11$ y 12 ). 
Figura 7: Resultados analíticos de consumo

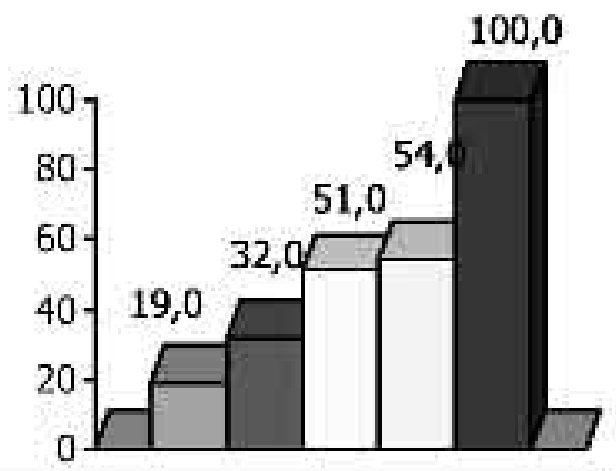

\begin{tabular}{ll|}
\hline Heroina & a Cocaína \\
$\square$ Cannabis & $\square$ Benzodiacepinas \\
nMeladona & \\
\hline
\end{tabular}

Figura 8: Autoinforme de consumo: Heroína

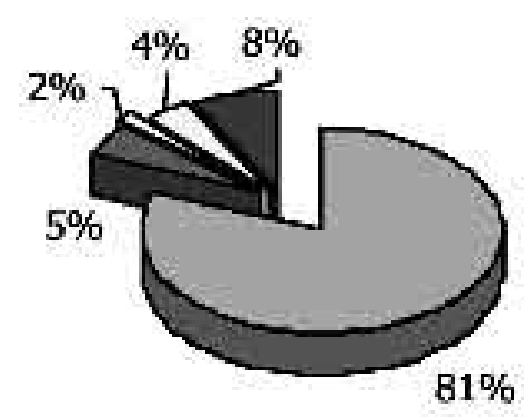

$$
\begin{aligned}
& \text { 口Aostinencia } \\
& \text { aMús } 2 \\
& \text { veces/mes } \\
& \text { a Más } 1 \\
& \text { vez/scmana } \\
& \text { a Más dos } \\
& \text { veces/5emana } \\
& \text { a varias } \\
& \text { veres/dia }
\end{aligned}
$$

Figura 9: Autoinforme de consumo: Cocaína

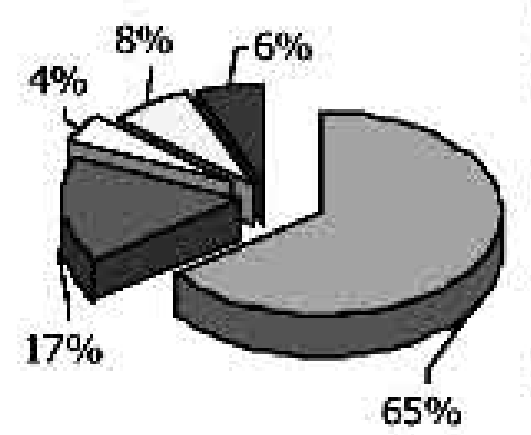

마stinencia

口Más 2

veces/mes

口Màs 1

wez/semaria

口Más dos veces/sernana

- Varias

veces/dia
Figura 10: Autoinforme consumo: Benzodiacepinas

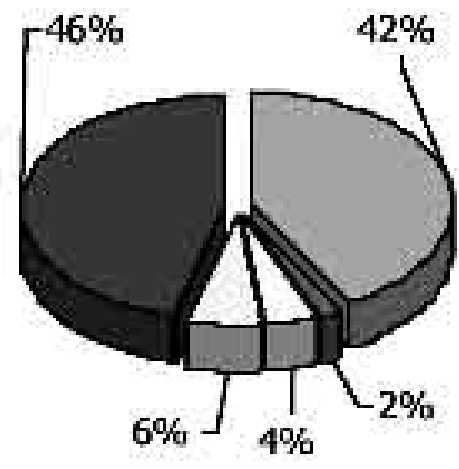

$\square$ Abstinencia

口Ma: Z

vecesirres

口MAis 1

vez/semana

口 Màs dos veces/semana

G Varias: wates/tía

Figura 11: Autoinforme de consumo: Cannabis

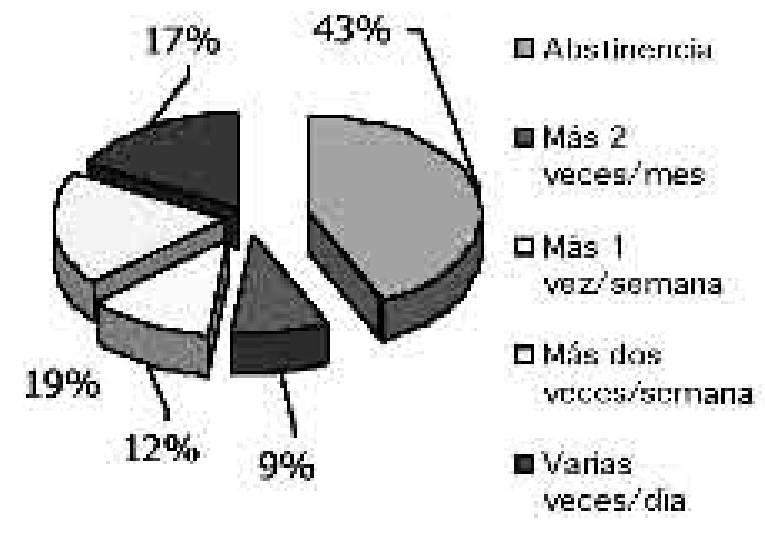

Figura 12: Autoinforme de consumo: Alcohol

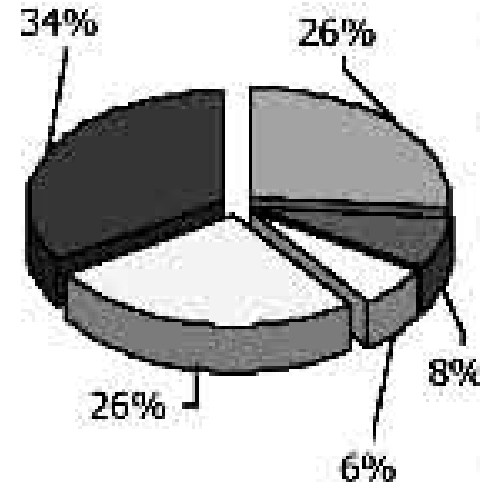

口Abstinencia

口 Má: 2

weces/mes

口Piss 1

vez/semiria

口 Más dos

veces/semana

Q Varia: verces/dia 


\section{Análisis bivariable}

Como podemos comprobar en esta tabla 1, las variables sociodemográficas consideradas apenas muestran relación con el grado de adhesión. Únicamente la situación legal actual se asocia a la adherencia semanal de baja exigencia, mostrando una tendencia a facilitar ésta en sujetos sin causas pendientes (tabla 1). El estadío CDC tampoco se ha relacionado con los niveles de adherencia.

Por lo que respecta al consumo de drogas, todos las variables consideradas muestran relación con algún nivel de adherencia, favoreciéndose ésta a partir de la abstinencia a cualquier sustancia. De manera más concreta, todos los indicadores analíticos de consumo se asocian a adherencia del día anterior, a adherencia semanal y a adherencia mensual de baja exigencia, mientras que en el caso de la adherencia mensual de alta exigencia únicamente los resultados positivos a cocaína y benzodiacepinas resultan significativos. El consumo evaluado a través de autoinforme repite la tendencias anteriores, aunque en algunos no llega al nivel de significación estadística (tabla 1).

En el análisis de varianza, todos los indicadores de adherencia se relacionan con el policonsumo, con mayor frecuencia del mismo, en el caso de los no adherentes. El otro resultado significativo señala que los sujetos adherentes considerados con la máxima exigencia llevaban más tiempo en tratamiento que aquellos no adherentes.

\section{Regresión logística}

La tabla 2 muestra los resultados de la regresión logística, con la ecuación de regresión para cada uno de los indicadores considerados y con el resumen final comparativo entre las predicciones hechas con la misma y la pertenencia real de los sujetos. En todos los casos, las ecuaciones de regresión configuradas están compuestas por dos variables, el policonsumo de heroína, cocaína y benzodiacepinas, que aparece en todas ellas, el autoinforme de consumo de cannabis para la adherencia del día anterior, semanal de alta y baja exigencia y mensual de baja exigencia, y el autoinforme de consumo de alcohol, primer predictor en el caso de la adherencia mensual de alta exigencia.

En cuanto a las predicciones, nuestras ecuaciones resultan más eficaces con los indicadores de baja exigencia y la adhesión del día anterior, llegando a clasificar al $75,26 \%$, al $74,23 \%$ y al $73,20 \%$ de los sujetos. Cuando estos indicadores se toman atendiendo al

\begin{tabular}{|c|c|c|c|c|c|c|c|c|c|c|}
\hline & \multicolumn{2}{|c|}{$\begin{array}{c}\text { AYER } \\
\text { (TOMA CORRECTA) }\end{array}$} & \multicolumn{2}{|c|}{$\begin{array}{c}\text { SEMANA } \\
\text { (ALTA EXIGENCIA) }\end{array}$} & \multicolumn{2}{|c|}{$\begin{array}{c}\text { SEMANA } \\
\text { (BAJA EXIGENCIA) }\end{array}$} & \multicolumn{2}{|c|}{$\begin{array}{c}\text { MES } \\
\text { (ALTA EXIGENCIA) }\end{array}$} & \multicolumn{2}{|c|}{$\begin{array}{c}\text { MES } \\
\text { (BAJA EXIGENCIA) }\end{array}$} \\
\hline & $\chi^{2}$ & $P$ & $\chi^{2}$ & $P$ & $\chi^{2}$ & $P$ & $\chi^{2}$ & $P$ & $\chi^{2}$ & $P$ \\
\hline Sexo & .252 & n.s. & .035 & n.s. & .063 & n.s. & .537 & n.s. & 0.33 & n.s. \\
\hline Nivel socioeconómico & .417 & n.s. & .330 & n.s. & 0.521 & n.s. & 1.362 & n.s. & .146 & n.s. \\
\hline Asistencia al centro & 5.745 & n.s. & 4.94 & n.s. & 5.11 & n.s. & 6.74 & n.s. & 6.861 & n.s. \\
\hline Situación legal anterior & 1.181 & n.s. & .175 & n.s. & 3.180 & n.s. & .135 & n.s. & 1.898 & n.s. \\
\hline Situación legal actual & 4.826 & n.s. & 4.651 & n.s. & 7.44 & .024 & .062 & n.s. & 4.397 & n.s. \\
\hline Nivel de estudios & 2.340 & n.s. & 1.882 & n.s. & 4.96 & n.s. & 1.271 & n.s. & 2.026 & n.s. \\
\hline Situación laboral & 5.882 & n.s. & 2.920 & n.s. & 6.83 & n.s. & 6.763 & n.s. & 6.359 & n.s. \\
\hline Convivencia actual & 4.024 & n.s. & 4.105 & n.s. & 4.041 & n.s. & 3.128 & n.s. & 3.757 & n.s. \\
\hline Analítica heroína & 6.867 & .009 & 5.177 & .023 & 8.93 & .003 & 3.107 & n.s. & 8.199 & .004 \\
\hline Analítica de cocaína & 8.569 & .003 & 8.425 & .004 & 6.28 & .012 & 6.335 & .012 & 10.599 & .001 \\
\hline Analítica cannabis & 5.974 & .015 & 6.807 & .009 & 7.36 & .007 & 3.726 & n.s. & 6.146 & .013 \\
\hline Analítica benzodiacepinas & 11.337 & .001 & 6.872 & .009 & 10.83 & .001 & 10.667 & .001 & 1.957 & .001 \\
\hline Autoinforme: heroína & 5.686 & n.s. & 4.35 & n.s. & 7.36 & n.s. & 6.841 & n.s. & 6.763 & n.s. \\
\hline Autoinforme: cocaína & 10.067 & .039 & 8.62 & n.s. & 9.250 & n.s. & 7.152 & n.s. & 12.120 & .016 \\
\hline Autoinforme: cannabis & 10.438 & .034 & 10.02 & .040 & 12.803 & .012 & 16.560 & .002 & 17.088 & .002 \\
\hline Autoinforme: benzos & 16.070 & .003 & 10.75 & .029 & 15.210 & .004 & 10.388 & .034 & 10.260 & .036 \\
\hline Autoinforme: alcohol & 11.579 & .021 & 8.54 & n.s. & 15.321 & .004 & 14.328 & .006 & 10.408 & .034 \\
\hline Estadío CDC $(A, B, C)$ & .683 & n.s. & 1.095 & n.s. & 1.785 & n.s. & .146 & n.s. & 1.214 & n.s. \\
\hline $\begin{array}{l}L_{\text {Linfocitos } C D_{4}} \\
\text { Carga viral indetectable }\end{array}$ & .451 & n.s. & .442 & n.s. & .106 & n.s. & 1.047 & n.s. & .226 & n.s. \\
\hline vs. detectable & 11.496 & .001 & 8.921 & .003 & 8.089 & .004 & 5.265 & .022 & 9.173 & .002 \\
\hline
\end{tabular}




\begin{tabular}{|c|c|c|c|c|c|c|c|}
\hline \multicolumn{8}{|c|}{ Variable criterio: AYERTOMA CORRECTA } \\
\hline Etapa & Predicto & & & & B & $\%$ Clas. & $\chi^{2}$ \\
\hline 1 & Policon: & no heroína, cocaí & a, benzodiace & & .8444 & 71.13 & 16.437 \\
\hline 2 & Autoinf & e de consumo $d$ & cannabis & & .3625 & 73.20 & 22.247 \\
\hline \multicolumn{8}{|c|}{ RESUMEN DE CLASIFICACIÓN } \\
\hline \multirow{3}{*}{\multicolumn{2}{|c|}{ Observaciones }} & Pronósticos & Adherentes & No adherentes & $\%$ clasi & & \multirow{3}{*}{$\begin{array}{c}\text { TOTAL } \\
\mathbf{7 3 . 2 0 \%}\end{array}$} \\
\hline & & Adherentes & 53 & 8 & 86.89 & & \\
\hline & & No adherentes & 18 & 18 & 50.00 & & \\
\hline \multicolumn{8}{|c|}{ Variable criterio: SEMANA BAJA EXIGENCIA } \\
\hline Etapa & \multicolumn{4}{|c|}{ Predictores } & B & $\%$ Clas. & $\chi^{2}$ \\
\hline 1 & \multicolumn{4}{|c|}{ Policonsumo heroína, cocaína, benzodiacepinas } & .8263 & $70.10 \%$ & 15.552 \\
\hline 2 & \multicolumn{4}{|c|}{ Autoinforme de consumo de cannabis } & .4254 & $74.23 \%$ & 23.138 \\
\hline \multicolumn{8}{|c|}{ RESUMEN DE CLASIFICACIÓN } \\
\hline \multirow{3}{*}{\multicolumn{2}{|c|}{ Observaciones }} & Pronósticos & Adherentes & No adherentes & $\%$ clasi & & \multirow{2}{*}{$\begin{array}{c}\text { TOTAL } \\
\mathbf{7 4 . 2 3 \%}\end{array}$} \\
\hline & & Adherentes & 55 & 9 & 85.94 & & \\
\hline & & No adherentes & 16 & 17 & 51.52 & & \\
\hline \multicolumn{8}{|c|}{ Variable criterio: SEMANA ALTA EXIGENCIA } \\
\hline Etapa & \multicolumn{4}{|c|}{ Predictores } & $\mathrm{B}$ & $\%$ Clas. & $\chi^{2}$ \\
\hline 1 & \multicolumn{4}{|c|}{ Policonsumo heroína, cocaína, benzodiacepinas } & .6995 & 68.04 & 12.48 \\
\hline 2 & \multicolumn{4}{|c|}{ Autoinforme de consumo de cannabis } & .33234 & 70.10 & 17.810 \\
\hline \multicolumn{8}{|c|}{ RESUMEN DE CLASIFICACIÓN } \\
\hline \multirow{3}{*}{\multicolumn{2}{|c|}{ Observaciones }} & Pronósticos & Adherentes & No adherentes & $\%$ clas & & \multirow{2}{*}{$\begin{array}{c}\text { TotAL } \\
\mathbf{7 0 . 1 0 \%}\end{array}$} \\
\hline & & Adherentes & 50 & 8 & 86.21 & & \\
\hline & & No adherentes & 21 & 18 & 46.15 & & \\
\hline & & Varia & ole criterio: M & AJA EXIGENCIA & & & \\
\hline Etapa & Predicto & & & & B & $\%$ Clas. & $\chi^{2}$ \\
\hline 1 & Policon & no heroína, cocaí & a, benzodiace & & .9361 & 73.20 & 18.909 \\
\hline 1 & Autoinf & ne de consumo de & cannabis & & .3818 & 75.26 & 24.935 \\
\hline & & & RESUMEN DE & SIFICACIÓN & & & \\
\hline & & Pronósticos & Adherentes & No adherentes & $\%$ clas & & TOtAL \\
\hline Obser & vaciones & Adherentes & 50 & 8 & 86.21 & & $70.10 \%$ \\
\hline & & No adherentes & 21 & 18 & 46.15 & & \\
\hline & & Vari & ble criterio: N & ILTA EXIGENCIA & & & \\
\hline Etapa & Predicto & & & & $\mathrm{B}$ & $\%$ Clas. & $\chi^{2}$ \\
\hline 1 & Autoinf & e de consumo de & alcohol & & .7425 & 69.07 & 14.090 \\
\hline 1 & Policon: & no heroína, cocaí & a, benzodiace & & .4750 & 70.10 & 23.791 \\
\hline & & & RESUMEN DE & SIFICACIÓN & & & \\
\hline & & Pronósticos & Adherentes & No adherentes & $\%$ clas & & TotAL \\
\hline Obser & vaciones & Adherentes & 28 & 15 & 65.12 & & $70.10 \%$ \\
\hline & & No adherentes & 14 & 40 & 74.07 & & \\
\hline
\end{tabular}


criterio de máxima exigencia, el porcentaje disminuye hasta el $70.10 \%$. Desarrollamos brevemente cada indicador, ya que, aún con porcentajes similares, no se comportan igual. Así, los dos indicadores de baja exigencia y del día anterior clasifican con bastante acierto a los sujetos adherentes (85.94\%, 87.30\% y 86,89\% respectivamente) y detectan a más de la mitad de los no adherentes (51,52\%, 52.94\%, 50,0\%). Para la adherencia semanal de alta exigencia las mismas variables siguen detectando a los adherentes, pero bajan ligeramente al clasificar a los no adherentes, únicamente al 46,15\%. Este pérdida de capacidad predictiva se debe a que, en este segundo caso, la ecuación considera como adherentes a sujetos que con el criterio de máxima exigencia no lo son.

Por último, el indicador de adherencia mensual de alta exigencia funciona de manera completamente diferente. Hay que tener en cuenta que la composición de los grupos de sujetos adherentes y no adherentes es bastante distinta al de los indicadores anteriores, ya que, ahora, consideramos como adherentes únicamente al 44,32 \% frente al 62,8\%, 65.9\%, 59.7\% y $64,9 \%$ del resto de grupos. No obstante, el porcentaje de casos correctamente clasificados es alto, el $70,10 \%$, acertando más con los no adherentes, el $74,07 \%$ son correctamente clasificados, que con los adherentes, el $65,12 \%$.

\section{DISCUSIÓN}

La principal conclusión que podemos obtener de este trabajo es que se pone claramente de manifiesto la influencia que el consumo de drogas tiene sobre la adherencia a los tratamientos antirretrovirales de los pacientes con VIH-SIDA en programa de tratamiento con metadona. Además, la relación entre consumo de drogas y adherencia se reproduce prácticamente para todos los tipos de drogas (heroína, cocaína, benzodiacepinas, alcohol y cannabis), para los dos métodos de evaluación de consumo (autoinforme y pruebas de orina) y para la mayoría de los indicadores de adherencia considerados (del día, de la semana y del mes anterior y con criterios de máxima y baja exigencia).

Estos datos vienen a apoyar los hallazgos encontrados en otros estudios en los que aparece la misma relación entre el consumo de distintas drogas, tanto ilegales como alcohol, y la no adherencia a los tratamientos antirretrovirales 17,16. Aunque en otro estudio con una muestra de población holandesa, sólo el $50 \%$ de los sujetos consumidores de drogas en activo reconocían no seguir la terapia antirretroviral ${ }^{18}$. En muestras españolas, encuentran relación entre baja adherencia y el hecho de haber consumido cocaína en los 9 meses anteriores a la recogida de datos ${ }^{13}$.
En nuestro estudio, y, a través del análisis multivariable, podemos ser más específicos al analizar la relación entre consumo y adherencia. En este sentido, hemos de destacar la importancia que tiene el policonsumo en la adherencia. Así, en los indicadores de adherencia del día anterior, de la semana anterior y del mes de baja exigencia, aparece el indicador compuesto de consumo de heroína, cocaína y benzodiacepinas como primer predictor, lo que viene a decir que los sujetos que consumen estas tres sustancias tienen menos probabilidad de ser buenos cumplidores que los que consumen dos y, éstos a su vez, menos que los sujetos que sólo consumen una droga. La importancia del policonsumo se pone de manifiesto al comprobar que la segunda y última variable de las ecuaciones de regresión mencionadas la constituye el consumo de cannabis. En el caso de la adherencia mensual de alta exigencia el consumo de alcohol y el indicador compuesto de consumo son igualmente los componentes de la ecuación de regresión.

Respecto a nuestros indicadores de adherencia, hemos de decir que no son directamente comparables a otros estudios que cuantifican la adherencia según el número de pastillas tomadas. No obstante, los porcentajes de adherencia encontrados no resultan especialmente distintos de otros estudios, aunque las comparaciones son difíciles de establecer por los pocos estudios existentes con población adicta, por las distintas características de las muestras y por las medidas usadas. Así, en la experiencia francesa, la comparación entre sujetos que habían adquirido el virus por ser ADVP y sujetos que lo habían adquirido a través de otras conductas de riesgo (relaciones sexuales) arroja tasas de adherencia similares en ambos grupos a los tres meses: $82,3 \%$ versus $83,2 \%$ respectivamente ${ }^{16}$. Mientras que, en la experiencia con pacientes suizos, aparecen porcentajes de adherencia algo más bajos, comparando a exconsumidores de drogas sin tratamiento con otros que están en tratamiento, 78,1\% versus $65,5 \%$ respectivamente a los tres meses ${ }^{19}$.

Cuando el plazo para evaluar la adherencia se amplia al año, la tasa de éxito se reduce notablemente tanto para los sujetos con antecedentes de consumo como para el resto, que en la experiencia canadiense, arrojan porcentajes del $30 \%$ y $43 \%$ respectivamente ${ }^{11}$. Aunque nuestra muestra no es directamente comparable con ninguna de las experiencias anteriores, ya que, incluye tanto a sujetos consumidores como no consumidores y con una gran variabilidad de tiempo de tratamiento, encontramos tasas de adherencia del 62\% para el día anterior y del 58\% para la última semana, que, según los datos anteriormente citados, resultan positivas al tratarse de sujetos que llevan una media de 6 años en programa de metadona, siendo, como ya hemos dicho antes, el consumo el principal factor determinante de la adherencia. 
Los resultados encontrados para la muestra madrileña reflejan que la población ex-consumidora de drogas presenta niveles de adherencia dentro de los rangos de otras poblaciones infectadas y similares también a los de cualquier otra enfermedad crónica. Las cifras que se manejan en los estudios llevados a cabo sobre el tema, son variables dependiendo de la enfermedad, del tipo de tratamiento, de los factores analizados y del método seguido. Ya en los estudios realizados en 1965 por Ley y Spelman se hablaba de que aproximadamente el $50 \%$ de los pacientes, de cualquier enfermedad estudiada, incumplían las recomendaciones médicas ${ }^{20}$. En pacientes con infección por $\mathrm{VIH}$, los autores hablan de un rango entre $46 \%$ y $88 \%$ de porcentaje de cumplidores ${ }^{21} \mathrm{y}$, en otras fuentes consultadas, se considera el porcentaje de pacientes adherentes, variable y dentro de un rango del 56\% al $83 \%$, para un cumplimiento de más del $80 \%$ de la toma de las dosis prescritas ${ }^{22}$.

En resumen, éstos datos nos ponen de relieve que existen grandes diferencias en los porcentajes de pacientes cumplidores con la terapia antirretroviral, siendo los rangos muy amplios al igual que lo son para otras enfermedades crónicas. También se contemplan diferencias dependiendo de la población de que se trate, ya que los resultados, en general, hablan de porcentajes más bajos de adherencia en cuanto se estudia una muestra de sujetos ADVP. No obstante, los porcentajes de adherencia de estos sujetos son más altos de lo que cabría esperar para una población tan inestable y con múltiples problemas psiquiátricos, no presentes en otros pacientes HIV+.

La única forma de evitar la aparición de resistencias es que el cumplimiento sea casi perfecto, algunos autores hablan del 95\%, e incluso, con este nivel de cumplimiento, sólo el $81 \%$ de los pacientes conseguirían mantener una carga vírica indetectable (menos de 50 copias por microlitro) ${ }^{23}$. Otros autores refieren que por debajo del $80 \%$ del cumplimiento ya se encuentra viremia indetectable en la mayoría de los pacientes ${ }^{24}$, mientras que otros ponen el límite en el $90 \%$ de las dosis prescritas debido a que los estudios revelan que, tras la introducción de los inhibidores de la proteasa, sólo por encima de este porcentaje de adherencia y respetando los intervalos entre dosis y la relación con las comidas, se obtienen resultados satisfactorios ${ }^{25}$.

En nuestro caso, hemos considerado cinco niveles de adherencia, con dos niveles de cumplimiento para el caso del mes y de la semana, alta y baja exigencia. En todos los casos hemos encontrado que hay una relación directa con la carga viral, pero no podemos establecer que en ninguno de nuestros indicadores se reduzca más la carga viral, ya que todos los estadísticos presentan valores similares.
Otro aspecto problemático hace referencia al método de medida de la adherencia. En nuestro caso hemos usado únicamente el autoinforme, pero podemos aportar datos indirectos a favor de este tipo de medidas si extrapolamos la experiencia obtenida con las muestras de orina y los autoinformes de consumo de drogas. La comparación de la información que los sujetos dan de su consumo de drogas durante la entrevista con el evaluador, con los datos de los controles toxicológicos realizados, refleja concordancias muy elevadas, lo que resulta llamativo en estos sujetos a los que habitualmente les cuesta mucho reconocer los retrocesos en su proceso de tratamiento y, por tanto, no suelen informar de los consumos de sustancias tóxicas, entre otros motivos por temor a las consecuencias negativas que de ello pudieran derivarse (legales, pérdida de privilegios en el CAID, pérdida de privilegios en sus casas, etc.). Quizás, podríamos explicar este dato por las características de nuestra muestra, con una larga estancia en tratamiento y con la posibilidad de establecer una buena relación con los profesionales que se convierten en una importante fuente de apoyo. Es posible que una vez lograda una buena relación terapéutica, se minimicen las consecuencias negativas del incumplimiento y se refuerce el papel activo del sujeto en el tratamiento, pudiendo servirnos del autoinforme del sujeto como indicador válido y suficiente de la adherencia al tratamiento.

En resumen, de nuestro estudio podemos concluir que los sujetos que están consumiendo drogas presentan una mayor probabilidad de abandonar la TARGA. De esta conclusión se pueden derivar algunas consideraciones relevantes para la práctica de los profesionales que intervienen directamente con los pacientes $\mathrm{VIH}$-SIDA que se encuentran en programas de deshabituación de su adicción a drogas.

Así, nos preguntarnos si no sería más conveniente retrasar el acceso a la TARGA de aquellos sujetos que se encuentren en periodo activo de consumo, ya que, el hecho de tener muchas posibilidades de abandonar el tratamiento acarrearía consecuencias negativas no sólo para su salud sino también para la de toda la comunidad, así como, un importante gasto sanitario.

Por otro lado, resulta imprescindible seguir trabajando con los sujetos con consumos activos de modo que puedan alcanzar la abstinencia y, posteriormente, acceder a la TARGA. Son muchos los trabajos publicados hasta la fecha en los que se demuestra que con una mínima intervención se consigue aumentar la adherencia al tratamiento antirretroviral de forma significativa con esta población ${ }^{16,26}$. Igualmente, hemos de resaltar la eficacia, ya demostrada, que para la mejor consecución de los éxitos en los tratamientos con metadona tiene la intervención psicosocial que se realiza desde los CAID27,28. 
Todo lo anterior exige una coordinación estrecha entre los diferentes niveles de atención que existen actualmente en España para esta población: hospital, CAID, PMM, centros de salud ambulatorios. Los CAID pueden trabajar la deshabituación y la abstinencia antes de derivar a un paciente a un centro hospitalario. Después pueden continuar con la labor educativa y realizar un control de la adherencia, prestando especial atención a aquellos sujetos con más dificultades para el cumplimiento (situación de calle, falta de apoyo social, trastornos psiquiátricos, consumo de otras drogas..), con los que se podrían aplicar medidas alternativas como la administración de la medicación desde el centro directamente (terapia directamente observada) y fomentar la formación de grupos de iguales, accesibles desde el centro, con las infinitas posibilidades que abren y que pueden aumentar el éxito de los tratamientos antirretrovirales ${ }^{29}$. Estas intervenciones se complementarían con las diseñadas desde los centros hospitalarios en los que se observa también un aumento de la adherencia con la intervención desde el servicio de farmacia (recuento de fármacos) y una mínima intervención psicosocial ${ }^{25,30}$.

Ya se ha demostrado que los métodos anteriores contribuyen a aumentar en mayor o menor grado la adherencia a los tratamientos antirretrovirales. Las investigaciones futuras deberían ir encaminadas a demostrar que los esfuerzos de estas intervenciones sirven para conseguir un grado de abandono menor de la TARGA, pudiéndose evaluar además el coste-efectividad y el coste-utilidad de estas intervenciones.

\section{REFERENCIAS}

1. Mocroft A, Johnson M, Phillips AN. Factors affecting survival in patients with the acquired immunodeficiency syndrome. AIDS 1996; 10: 1057-1065.

2. Gulick R, Mellors J, Harlir D, Eron J, Gonzalez C, Mc Mahon $D$ et al. Potent and sustained antiretroviral activity on indinavir, in combination with zidavudine and lamivudine. III Conference on Retrovirus and opportunistic infections. Washington, 1996.

3. Mathez D, Truchis P, Gorin I, Katlama C, Pialoux G, Saimot $A G$, et al. Ritonavir, AZT, DDC as a triple combination in AIDS patients. III Conference on Retrovirus and opportunistic infections. Washington, 1996.

4. Hammer SM, Yeny P. Antiretroviral therapy: Where are we?. AIDS 1998; 12: 181-188.

5. Lange JM. Changing therapy in HIV. AIDS 1996; 10 Suppl 1: 27-30.

6. Haynes RB, Sackett DL, Taylor DW. Compliance in health care. Baltimore: The Johns Hopkins University Press; 1979.
7. Ballester R, Reinoso I, García S, Campos A. Adherencia al tratamiento en la infección por VIH. Análisis y modificación de conducta 2000; 26: 689-716.

8. Haynes RB. Strategies to improve compliance with referral appointments and prescribed medical regimens. En RB Haynes, DL Sackett, DW, Taylor (Eds) Compliance in heath care. Baltimore: The Johns Hopkins University Press; 1979. p 121-143.

9. DiMatteo MR, Linn LS, Chang BL, Cope DW. Affect and neutrality in physician behavior: A study of patients, values and satisfaction. Journal of Behavioral Medicine 1985; 8: 397-409.

10. Rodríguez-Marín, J. Psicología social de la salud. Madrid: Síntesis; 1995.

11. Wood E, Montaner JSG, Braitstein P, Yip B, Schecter MT, O'Shaughnessy MVO, et al. Elevated rates of antirretroviral treatment discontinuation among HIVinfected injection drug users: implications for drug policy and public health. International Journal of Drug Policy 2004; 15: 133-138.

12. Gordillo V, del Amo J, Soriano V, González-Lahoz J. Sociodemographic and psychological variables influencing adherence to antirretroviral therapy. AIDS 1999; 13: 1763-1769.

13. Riera M, De La Fuente L, Castanyer B, Puigventós F, Villalonga C, Ribas MA, et al. Adherencia a los fármacos antirretrovirales medida por la concentración de fármacos y el recuento de comprimidos. Variables relacionadas con una mala adherencia. Med Clin 2002; 119: 286-92.

14. Hammer S, Katzenstein D, Hughes M, Gundacker $\mathrm{H}$, Schooley RT, Haubrich $\mathrm{RH}$, et al. A trial comparing nucleoside monotherapy with combination therapy in HIV-infected adults with $\mathrm{CD}_{4}$ cell counts from 200 to 500 per cubic milimeter. New England Journal of Medicine 1996; 335: 1081-1090.

15. loannidis J, Bassett R, Hughes M, Volberding P, Sacks $\mathrm{H}$, Lau J. Predictors and impact of patients lost to follow-up in a long-term randomized trial of immediate versus deferred antiretroviral treatment. J Acquir Immune Defic Syndr Hum Retrovirol 1997; 16: 22-30.

16. Moatti JP, Carrieri M, Spire B, Gastaut J, Cassuto J, Moreau J. Adherence to HAART in French HIV-infected injecting drug users: the contribution of buprenorphine drug maintenance treatment. The Manif 2000 study group. AIDS 2000; 14: 151-155.

17. Haubrich R, Little S, Currier J, Forthal D, Kemper C, Beall $G$, et al. The value of patient-reported adherence to antiretroviral therapy in predicting virologic and immunologic response. AIDS 1999; 13: 1099-1107.

18. Witteveen E, Van Ameijden E. Drug users and HIVCombination therapy (HAART): Factors which impede or facilitate adherence. Substance use \& misuse 2002; 37:1905-1925. 
19. Broers B, Morabia A, Hirschel B. A cohort study of drug users' compliance with zidovudine treatment. Archives of Internal Medicine 1994; 154: 1121-1127.

20. Ley P, Spelman MS. Communicating with the patient. London: Staple press; 1967.

21. Pedreira Andrade JD. 1999. Adherencia al tratamiento antiretrovírico del paciente infectado por $\mathrm{VIH}$. Publication of SEISIDA 1999; 10: 167-168.

22. Metha S, Moore RD, Graham NMH. Potential factors affecting adherence with HIV therapy. AIDS 1997; 11: 1665-1670.

23. Remor E. Valoración de la adhesión al tratamiento antirretroviral en pacientes HIV positivos. Psicothema 2002; 14: 262-267.

24. Chesney MA. Factors affecting adherence to antiretroviral therapy. Clin Infect Dis 2000; 30 Supl 2: 171-176.

25. Knobel H, Codina C, Miró JM, Carmona A, García B, Antela A, et al. Recomendaciones GESIDA/SEFH/PNS para mejorar la adherencia al tratamiento antirretroviral. Enfermedades Infecciosas y Microbiología Clínica 2000; 18: 27-39.

26. Malow R, McPherson S, Klimas N, Antoni M, Schneiderman N, Penedo $F$, et al. Adherence to
Complex Combination Antiretroviral Therapies by HIVPositive Drug Abusers. Psychiatric Services 1998; 49: 1021-1022.

27. Fernández JJ, González MP, Sáiz PA, Gutierrez E, Bobes J. Calidad de vida y severidad de la adicción en heroinómanos en tratamiento prolongado con metadona. Adicciones 1999; 11: 43-52.

28. Serrano M, Arrizabalaga I. Programa de mantenimiento con metadona. Una intervención multidisciplinar y comunitaria: un estudio de calidad de vida. En V Encuentro Nacional sobre drogodependencias y su enfoque comunitario. Cádiz 1998, p.135-142.

29. Broadhead RS, Heckathorn DD, Altice FL, Van Hulst $\mathrm{Y}$, Carbone M, Friedland $\mathrm{GH}$, et al. Increasing drug users' adherence to HIV treatment: results of a peerdriven intervention feasibility study. Social Science and Medicine 2002; 55: 235-246.

30. Luber A. Community collaborations between physicians and pharmacists improved adherence with HIV Consensus Panel Guidelines and enhances the care of HIV infected individuals. En $40^{\text {th }}$ Interscience Conference on Antimicrobial Agents and Chemotherapy. Toronto, Canadá 2000 [abstract 800]. 